\title{
The effect of acute feeding of carnitine, acetyl carnitine and propionyl carnitine on basal and A23187-stimulated eicosanoid release from rat carrageenan-elicited peritoneal macrophages
}

\author{
BY G. R. ELLIOTT*, A. P. M. LAUWEN AND I. L. BONTA \\ Pharmacology Department, Erasmus University Rotterdam, Postbox 1738, 3000DR, \\ Rotterdam, The Netherlands
}

(Received 18 July 1989 - Accepted 30 March 1990)

Little is known about the ability of carnitine to modulate cell functions. As carnitine plays an important role in lipid metabolism we investigated the acute effect of $L$-carnitine, $L$-acetyl carnitine and $L$-propionyl carnitine $(300 \mathrm{mg} / \mathrm{kg}$ per $\mathrm{d} ; 4 \mathrm{~d}$ ) on the basal and calcium-ionophore (A23187)-stimulated release of arachidonic acid metabolites from rat carrageenan-elicited peritoneal macrophages. $A$ decrease in the number of peritoneal carrageenan-elicited macrophages was observed after feeding all three compounds. The basal release of prostaglandin $E_{2}, 6$ keto-prostaglandin $F_{1 \alpha}$ and leukotriene $B_{4}$ was stimulated by all treatments. In contrast, thromboxane $B_{2}$ production was diminished by feeding carnitine and acetyl carnitine. A23187-stimulated synthesis of 6 keto-prostaglandin $F_{1 z}$ and leukotriene $B_{4}$ was further enhanced by all three compounds. Acetyl carnitine and propionyl carnitine also enhanced thromboxane $B_{2}$ synthesis. However, no effects on prostaglandin $E_{2}$ formation were detected. The 6 keto-prostaglandin $F_{1 \alpha}$ : thromboxane $B_{2}$ ratio, calculated from the basal and A23187-stimulated values, was increased by carnitine treatment. In the presence of $\mathbf{A 2 3 1 8 7}$ there was also an increase in the 6 keto-prostaglandin $F_{b a}$ : leukotriene $B_{4}$ ratio. We conclude that carnitine, and possibly some of its derivatives, could modify the macrophage component of an inflammation in vivo.

Carnitine compounds: Peritoneal macrophages : Arachidonic acid metabolites: Rat

L-Carnitine ( $\beta$-hydroxy- $(\gamma-N$-trimethylamino $)$-butyrate) is a natural amino acid, found particularly in animal cells, which plays an important role in fatty acid (FA) metabolism. Its major function appears to be the transport of long-chain fatty acids into mitochondria for oxidation, particularly in the heart and skeletal muscles. It has also been suggested that under abnormal metabolic conditions, e.g. diabetes, carnitine could act as a buffer for excess organic acids, so protecting mitochondrial integrity. Its most important medical use is in treating conditions characterized by a carnitine deficiency, resulting from either a genetic defect or medical intervention, e.g. an operation or renal dialysis (Bremer, 1983). It has also been suggested that carnitine supplements may be useful in reversing Intralipid ${ }^{\mathbb{R}}$ induced immunosuppression (De Simone et al. 1982).

Recently carnitine has been shown to modify lipid metabolism in a way which indicates that it could play a more general regulatory role than was previously thought. Specifically, carnitine has been shown to enhance the formation of arachidonic acid (AA) from linoleic acid by isolated hepatocytes (Christopherson \& Norseth, 1981). Cyclooxygenase (CO) and lipoxygenase (LO) metabolites of AA (eicosanoids) are important local modulators of inflammations. In general the $\mathrm{CO}$ metabolites are anti- and the LO metabolites proinflammatory, and their effects on a target cell or tissue are often opposing. For example,

* Present address: Pharmacology Department, Medical Biological Laboratory, TNO, PO Box 45, 2280 AA, Rijswijk ZH, The Netherlands. 
prostaglandin $(\mathrm{PG}) \mathrm{E}_{2}$, a CO product, inhibits and leukotriene (LT)C $\mathrm{C}_{4}$, a LO metabolite, stimulates macrophage lysosomal enzyme secretion and formation of active oxygen species (Smith \& Weidemann, 1980; Schenkelaars \& Bonta, 1986). The macrophage is both a target for and a source of eicosanoids. Besides $\mathrm{PGE}_{2}$ and $\mathrm{LTC}_{4}$, macrophages also release $\mathrm{PGI}_{2}$ (prostacyclin) and thromboxane (TX)A $\mathrm{A}_{2}$, CO metabolites, and LTB $\mathrm{L}_{4}$, an LO product. These eicosanoids also possess biological activities. LTB $_{4}$ is a contractor of smooth muscle, increases blood vessel permeability, is chemotactic for neutrophils and promotes neutrophil aggregation and adherence to endothelial cells. Similarly, $\mathrm{TXA}_{2}$ is a strong contractor of smooth muscle (lung and blood vessels) and promotes platelet aggregation. Thus these two eicosanoids play an important role in vascular inflammations. In contrast, $\mathrm{PGI}_{2}$ relaxes smooth muscle and inhibits both platelet and neutrophil aggregation and neutrophil adherence to vascular endothelial cells (Ford-Hutchinson et al. 1980; Vargaftig et al. 1981). Thus, alterations in the release of the different products at the site of an inflammation could result in changes in the course of the inflammation. Interestingly, carnitine has been reported to inhibit human neutrophil superoxide production, a reaction also known to be modified by eicosanoids (Schinetti \& Mazzini, 1986). As a first step in evaluating the possibility that carnitine could influence eicosanoid synthesis we investigated the effect of feeding L-carnitine, L-acetyl carnitine (formed during $\beta$-oxidation of even-chain fatty acids) and $\mathrm{L}$-propionyl-carnitine (formed during $\beta$-oxidation of uneven-chain fatty acids) on the basal and ionophore (A23187)-stimulated synthesis and release of $\mathrm{PGE}_{2}, \mathrm{PGI}_{2}$ (assayed as 6 keto- $\mathrm{PGF}_{1 \alpha}$ ), $\mathrm{TXA}_{2}$ (assayed as $\mathrm{TXB}_{2}$ ) and $\mathrm{LTB}_{4}$ from macrophages ex vivo. Our results showed that carnitine modified macrophage eicosanoid synthesis and decreased the ability of the cell to respond to chemotactic stimuli.

\section{MATERIALS AND METHODS}

Feeding regimen

Male Wistar rats (16 weeks of age, four rats/group) were given $300 \mathrm{mg}$ carnitine or carnitine equivalent (acetyl carnitine and propionyl carnitine) $/ \mathrm{kg}$, dissolved in $1 \mathrm{ml}$ distilled water, by intubation on days $1-4$. Control animals were given distilled water.

\section{Isolation and incubation of peritoneal macrophages}

On day 1 all animals were injected with $2 \mathrm{ml}$ of a carrageenan solution $(1 \mathrm{mg} / \mathrm{ml})$ intraperitoneally. On day $4,1 \mathrm{~h}$ after the last administration of carnitine(s), the macrophages were isolated from pooled Gey's balanced-salt solution washes of the peritonea by density-gradient centrifugation over Lymphoprep (Nyegaard Diagnostica, Postbox 4220, Torshov, 0401 Oslo 4, Norway) (Schenkelaars \& Bonta, 1986) and suspended in Dulbecco's modification of Eagle's medium (DMEM) $\left(2 \times 10^{6}\right.$ nucleated cells $/ \mathrm{ml}$ ). Portions $(1 \mathrm{ml})$ of the macrophage preparation were incubated at $37^{\circ}$ for either $2 \mathrm{~h}$ (basal release) or $30 \mathrm{~min}$ with $10^{-6} \mathrm{M}-\mathrm{A} 23187$ (ionophore-stimulated release). These time periods were found to be optimal during pilot experiments. The cells were then centrifuged and the supernatant fractions analysed for $\mathrm{TXB}_{2}, \mathrm{PGE}_{2}, 6$ keto-PGF $\mathrm{PG}_{1 \alpha}$ and $\mathrm{LTB}_{4}$ by radioimmunoassay (Zijlstra \& Vincent, 1984). L-Lactate dehydrogenase (LDH) $(E C 1.1 .1 .27)$ activity was assayed using the method of Wroblewsky \& LaDue (1955).

The in vitro effect of carnitine on macrophage eicosanoid release

Macrophages $\left(2 \times 10^{6} / \mathrm{ml}\right.$ DMEM) were incubated with carnitine for $2 \mathrm{~h}$ or $24 \mathrm{~h}\left(37^{\circ}, 5 \%\right.$ carbon dioxide) in $55 \mathrm{~mm}$ petri dishes. The supernatant fractions were then collected and analysed for eicosanoids and $\mathrm{LDH}$. 


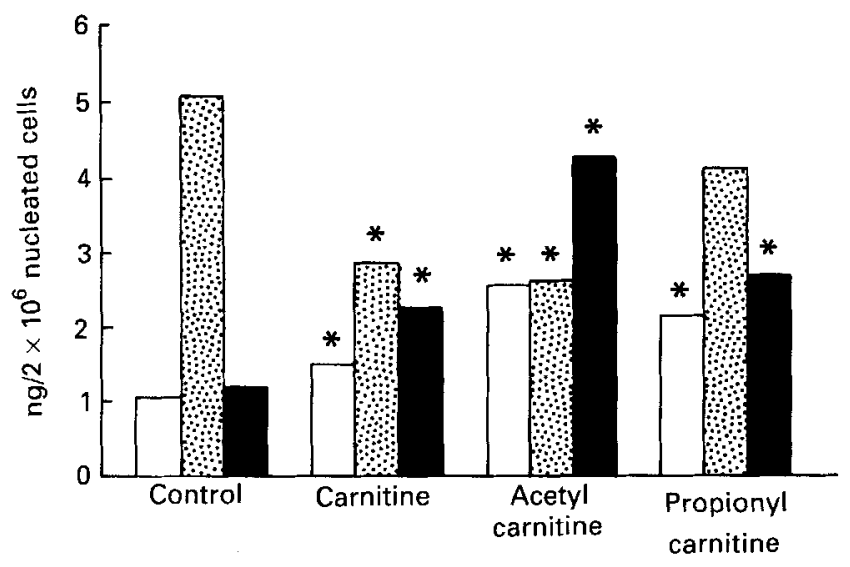

Fig. 1. The effect of feeding carnitine or its derivatives on the basal release of cyclooxygenase metabolites, prostaglandin $E_{2}(\square)$, thromboxane $B_{2}$ (圆) and 6 keto-prostaglandin $F_{k x}(a)$, from carrageenan-elicited peritoneal macrophages. Results are mean values, $n>9$. Mean values were significantly different from control values (Mann-Whitney $U$ test): ${ }^{*} P<0.005$.

\section{Statistics}

The feeding experiments were performed three times. Basal and A23187-stimulated release experiments were carried out at least in triplicate. Unless otherwise stated the values given are the mean values for each point $(n 10-12)$. Statistical significance was calculated using the two-tailed Mann-Whitney U test.

\section{RESULTS}

There were no differences in body-weights between the different groups. None of the carnitine compounds effected the release of $\mathrm{LDH}$ (values not given).

\section{The effect of feeding on carrageenan-induced peritoneal macrophage accumulation}

All three compounds significantly $(P<0.005)$ reduced by about half the number of macrophages isolated from peritonea $4 \mathrm{~d}$ after an intraperitoneal injection of carrageenan $\left(\times 10^{6} ; n 3\right)$ : control 40 (SD 11), carnitine 19 (SD 6), acetyl carnitine 14 (SD 7), propionyl carnitine $19 \cdot 4$ (SD 4).

The effect of feeding on the basal and A23187-stimulated release of eicosanoids from carrageenan-elicited rat peritoneal macrophages

The basal synthesis and release of $\mathrm{PGE}_{2}, 6$ keto-PGF $\mathrm{PG}_{1 x}$ and $\mathrm{LTB}_{4}$ were stimulated by all three treatments. In contrast to the effect on other eicosanoids, $\mathrm{TXB}_{2}$ formation was inhibited (carnitine and acetyl carnitine) or not modified (propionyl carnitine) (Fig. 1, Table 1). As a result the 6 keto- $\mathrm{PGF}_{1 \alpha}: \mathrm{TXB}_{2}$ ratio increased (Table 2). A23187 stimulated the release of the four eicosanoids assayed. Synthesis of 6 keto-PGF ${ }_{1 \alpha}$ and LTB $_{4}$ was further enhanced by all three carnitines, and acetyl carnitine and propionyl carnitine treatments were also associated with an enhanced $\mathrm{TXB}_{2}$ synthesis. There was no effect on $\mathrm{PGE}_{2}$ formation (Fig. 2, Table 1). The stimulation of 6 keto- $\mathrm{PGF}_{1 \alpha}$ release from macrophages isolated from carnitine-treated rats, together with the lack of effect on $\mathrm{TXB}_{2}$ synthesis, meant that the 6 keto- $\mathrm{PGF}_{1 \alpha}: \mathrm{TXB}_{2}$ ratio again increased. This was not the case with acetyl or propionyl carnitine. Furthermore, carnitine treatment also resulted in an increase in 6 keto-PGF ${ }_{1 \alpha}$ relative to $\mathrm{LTB}_{4}$ (Table 2). 
Table 1. The effect of carnitine or its derivatives on the basal and A23187-stimulated release of leukotriene $B_{4}\left(L T B_{4}\right)$ from carrageenan-elicited rat peritoneal macrophages

(Values are means of three experiments; $n>9$ )

\begin{tabular}{|c|c|c|c|c|}
\hline \multirow[b]{2}{*}{ Treatment } & \multicolumn{4}{|c|}{$\begin{array}{l}\text { LTB }_{4} \text { synthesis and release } \\
\left(\mathrm{ng} / 2 \times 10^{6} \text { nucleated cells) }\right.\end{array}$} \\
\hline & Control & Carnitine & $\begin{array}{l}\text { Acetyl } \\
\text { carnitine }\end{array}$ & $\begin{array}{l}\text { Propionyl } \\
\text { carnitine }\end{array}$ \\
\hline Basal release & 0.065 & $0 \cdot 104^{*}$ & $0 \cdot 143^{*}$ & $0 \cdot 116^{*}$ \\
\hline A23187-stimulated release & $0 \cdot 838$ & $1.644^{*}$ & $1.857^{*}$ & $1 \cdot 308^{*}$ \\
\hline
\end{tabular}

Mean values were significantly different from the corresponding control values (Mann-Whitney $U$ test): * $P<0-005$.

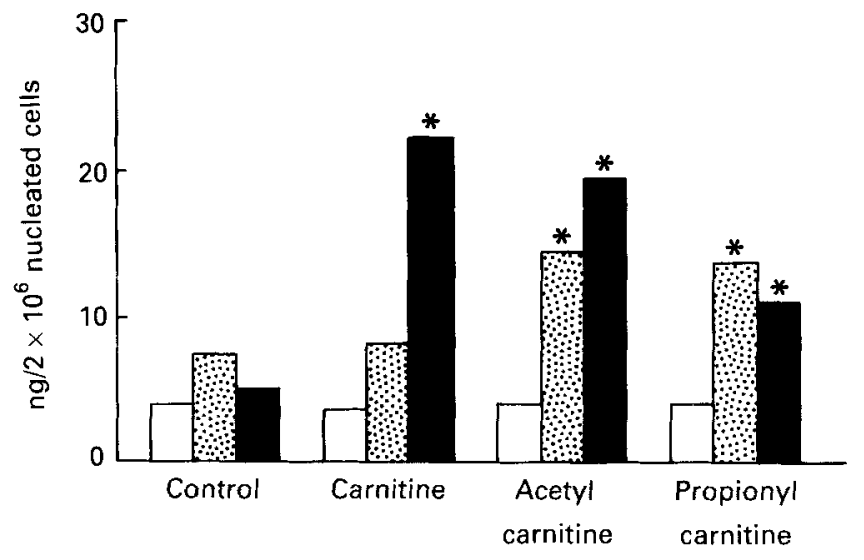

Fig. 2. The effect of feeding carnitine or its derivatives on the A23187-stimulated release of cyclooxygenase metabolites, prostaglandin $E_{2}(\square)$, thromboxane $B_{2}(\square)$ and 6 keto-prostaglandin $F_{1 \alpha}(\square)$ from carrageenanelicited peritoneal macrophages. Results are mean values, $n>9$. Mean values were significantly different from control values (Mann-Whitney U test): * $P<0.005$.

Table 2. The effect of feeding carnitine or its derivatives on the 6 keto-prostaglandin $(P G)$ $F_{1 x}$ :thromboxane $(T X) B_{2}$ and 6 keto- $P G F_{1 \alpha}$ :leukotriene $(L T) B_{4}$ ratios using the basal and A23187-stimulated release of arachidonic acid metabolites from carrageenan-elicited peritoneal macrophages

(Values are means of each experiment; $n>9$ )

\begin{tabular}{|c|c|c|c|c|}
\hline Treatment & Control & Carnitine & $\begin{array}{c}\text { Acetyl } \\
\text { carnitine }\end{array}$ & $\begin{array}{l}\text { Propionyl } \\
\text { carnitine }\end{array}$ \\
\hline $\begin{array}{l}6 \text { keto-PGF }{ }_{1 x}: \mathrm{TXB}_{2} \\
\text { Control } \\
\quad+\mathrm{A} 23187\end{array}$ & $\begin{array}{l}0.22 \\
1.02\end{array}$ & $\begin{array}{l}0.86^{*} \\
4 \cdot 95^{*}\end{array}$ & $\begin{array}{l}1 \cdot 57^{*} \\
1 \cdot 36\end{array}$ & $\begin{array}{l}1.00^{*} \\
0.82\end{array}$ \\
\hline $\begin{array}{l}6 \text { keto-PGF } 1 \alpha: \mathrm{LTB}_{4} \\
\text { Control } \\
\quad+\mathrm{A} 23187\end{array}$ & $\begin{array}{r}21 \cdot 35 \\
6 \cdot 70\end{array}$ & $\begin{array}{l}24 \cdot 70 \\
15 \cdot 19^{*}\end{array}$ & $\begin{array}{l}45 \cdot 4 \\
10 \cdot 78\end{array}$ & $\begin{array}{r}24 \cdot 40 \\
9 \cdot 14\end{array}$ \\
\hline
\end{tabular}

Mean values were significnatly different from the control values (Mann-Whitney $U$ test): ${ }^{*} P<0.005$. 
The effect on eicosanoid synthesis of incubating macrophages in vitro with carnitine We could detect no effect of carnitine on macrophage eicosanoid or LDH release during incubations of $2 \mathrm{~h}$ or $24 \mathrm{~h}$ (values not shown).

\section{DISCUSSION}

The results presented here clearly show that the acute oral administration of carnitine and two of its derivatives modified the ability of macrophages to synthesize eicosanoids and to respond to chemotactic signals generated when carrageenan was injected intraperitoneally. We found that the basal release of three of the four eicosanoids assayed, $\mathrm{PGI}_{2}, \mathrm{PGE}_{2}$ and LTB $_{4}$, was enhanced by all three carnitine compounds, whereas TXA $_{2}$ production was inhibited by carnitine and acetyl carnitine. In general carnitine compounds also enhanced A23187-stimulated eicosanoid synthesis. $\mathrm{PGI}_{2}$ and $\mathrm{LTB}_{4}$ formation were enhanced by carnitine, acetyl carnitine and propionyl carnitine treatment, and $\mathrm{TXA}_{2}$ formation by acetyl carnitine and propionyl carnitine. $\mathrm{PGE}_{2}$ production was not modified. It is not clear what mechanisms are involved. No changes in $\mathrm{LDH}$ release were detected ex vivo or after incubating macrophages with carnitine in vitro, so it would appear unlikely that the carnitine compounds were acting indirectly by damaging the macrophages. Furthermore, carnitine had no effect on eicosanoid synthesis in vitro over $2 \mathrm{~h}$ or $24 \mathrm{~h}$ incubation periods. AA has to be released from phospholipid by the action of a phospholipase (PLA or PLC) $^{2}$ before being metabolized to eicosanoids (Levine, 1982). After activation an increase in macrophage eicosanoid release is usually detected within $30 \mathrm{~min}$ (Gemsa et al. 1982). It is, therefore, unlikely that the alterations in eicosanoid synthesis are due to a stimulation of $\mathrm{PLA}_{2}$. A direct stimulation of CO or LO enzymes would also appear to be unlikely. Carnitine can stimulate FA synthesis by removing the inhibitory effect of long-chain acyl coenzyme $\mathrm{A}(\mathrm{CoA})$ on acetyl $\mathrm{CoA}$ carboxylase (EC 6.4.1.2), and Christopherson \& Norseth (1981) suggested that carnitine could stimulate FA chain elongation, and hence AA formation, via a similar mechanism. We incubated the macrophages with carnitine in vitro in DMEM without fetal calf serum or added FA. It is conceivable, therefore, that macrophage lipid turnover was too low for AA synthesis to be affected by carnitine. Further experiments, in the presence of exogenous FA, are needed before this proposal can be properly assessed. The finding that treatment with carnitine(s) had differential effects on eicosanoid release is of interest. Not all stimuli of AA turnover enhance both $\mathrm{CO}$ and $\mathrm{LO}$ metabolism. A23187, for example, stimulates the $\mathrm{LO}$ and $\mathrm{CO}$; phorbol esters only stimulate the $\mathrm{CO} ; \gamma$-hexachlorocyclohexane the $\mathrm{LO}$; and $\mathrm{LTC}_{4}$ stimulates $\left(\mathrm{PGE}_{2}\right)$ and inhibits $\left(\mathrm{TXA}_{2}\right.$ ) synthesis of different CO metabolites (Humes et al. 1982; Mead et al. 1984; Schenkelaars \& Bonta, 1986). These findings have been interpreted as showing different pools of AA which are metabolized via one or the other pathway. This concept is further supported by the findings presented here. However, it is the first time, to our knowledge, that a compound has been shown to stimulate elicited macrophage $\mathrm{PGI}_{2}$ synthesis in preference to $\mathrm{PGE}_{2}$.

As mentioned previously, treatment of rats with the test compounds resulted in a stimulation of the basal release of three of the four eicosanoids assayed. Two of these, $\mathrm{PGE}_{2}$ and $\mathrm{PGI}_{2}$, are considered to possess anti-inflammatory properties, whilst $\mathrm{LTB}_{4}$ and $\mathrm{TXA}_{2}$ are considered to be pro-inflammatory. Changes in eicosanoid ratios induced by feeding carnitine(s) could have important implications for the regulation of inflammatory events in general, particularly if carnitine also modifies eicosanoid release from cells other than macrophages. It has become apparent over the last few years that the relative concentrations of the different eicosanoids are important in regulating expression of various cell or tissue activities. Stimulation by $\mathrm{LTC}_{4}$ of macrophage cytostasis against tumour cells (Ophir et al. 1987) and LT stimulation of fibroblast division (Baud et al. 1987), 
for example, are enhanced by CO inhibitors, and Ophir ef al. (1987) suggested that the full

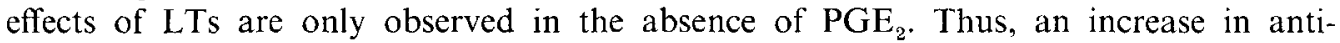
inflammatory $\mathrm{CO}$ metabolites would be expected to limit cell stimulation by LTs and reduce the effect of inflammatory mediators that depend on a functioning LO for full expression of their activities, e.g. interleukin-1 (Kunkel \& Chensue, 1985). This concept is supported by the finding that cell infiltration into the peritoneum, in response to chemotactic stimuli induced by carrageenan, was reduced by carnitine(s) treatment. This fact, together with the report of Schinetti \& Mazzini (1986) that carnitine inhibits neutrophil superoxide production, suggests that carnitine(s) could be anti-inflammatory in vivo.

A decrease in the $\mathrm{PGI}_{2}: \mathrm{TXA}_{2}$ ratio is thought to be an important factor in the progression of some cardiovascular diseases (Gryglewski et al. 1978). The $\mathrm{PGI}_{2}$ : $\mathrm{TXA}_{2}$ ratio (basal release) increased for all compounds tested. It is possible that carnitine(s) also modifies endothelial cell and platelet eicosanoid synthesis in a manner similar to that observed for macrophages, i.e. an increase in endothelial cell $\mathrm{PGI}_{2}$ and decrease in platelet $\mathrm{TXA}_{2}$. In that case both the macrophage and vascular components of an inflammation could be depressed. However, further experiments are needed before we can realistically assess the therapeutic potential of carnitine as an anti-inflammatory drug.

When evaluating the absolute changes in eicosanoid synthesis it must be borne in mind that the total number of cells recovered from treated animals was only about $50 \%$ of those isolated from controls. Thus, although $\mathrm{PGI}_{2}$ synthesis per $2 \times 10^{6}$ cells was stimulated by carnitine treatment the absolute $\mathrm{PGI}_{2}$ concentration in vivo could be the same as that of control animals due to the reduced number of infiltrating cells. Conversely, concentrations of TXA $_{2}$ in vivo would be much less than control values due to $(a)$ the inhibition of its synthesis and $(b)$ the reduction in peritoneal cell number.

In summary we have shown, for the first time, that the acute oral administration of carnitine and some of its derivatives can modify macrophage functions; eicosanoid release, particularly that of $\mathrm{PGI}_{2}$, and migration or chemotaxis. Although the significance in vivo of these findings with respect to modulation of an inflammatory disease has yet to be demonstrated, it is possible that carnitine supplements could be used for treating a wider range of conditions than carnitine deficiency syndromes.

The authors wish to thank Sigma-Tau Pharmaceutical Co., Rome, for financing this research.

\section{REFERENCES}

Baud, L., Perez, P., Denis, M. \& Ardaillou, R. (1987). Modulation of fibroblast growth by sulfidopeptide leukotrienes: effect of indomethacin. Joumal of Immunology 138, $1190-1195$.

Bremer, J. (1983). Carnitine: metabolism and functions. Physiological Review 63, 1420-1480.

Christopherson, B. O. \& Norseth, J. (1981). Arachidonic acid synthesis studied in isolated liver cells. Effects of (-)-carnitine and of (+)-decanoylcarnitine. FEBS Letters 133, 201204.

De Simone, C., Ferrari, M., Lozzi, A., Meli, D., Ricca, D. \& Sorice, F. (1982). Vitamins and immunity. II. influence of L-carnitine on the immune system. Acta Vitaminologica et Enzymologica 4, 135-140.

Ford-Hutchinson, A. W., Bray, M. A., Doig, D. V., Shipley, M. E. \& Smith, M. J. H. (1980). Leukotriene B , a $^{2}$ potent chemotactic and aggregating substance released by polymorphonuclear leucocytes. Nature 286, $264-265$.

Gemsa, D., Leser, H. G., Seitz, M., Debatin, M., Barlin, E., Deimann, W. \& Kramer, W. (1982). Cells in inflammation: the role of macrophages in inflammation. In Agents and Actions Supplements, vol. 11, pp. 93-114 [M. J. Parnham and J. Winkelmann, editors]. Basel, Boston and Stuttgart: Birkhauser-Verlag.

Gryglewski, R. J., Dembinska-Kiec, A., Zmuda, A. \& Gryglewska, T. (1978). Prostacyclin and thromboxane A. biosynthesis capacities of heart, arteries and platelets at various stages of experimental atherosclerosis in rabbits. Atherosclerosis 31, 385-394.

Humes, J. L., Burger, S., Galavage, M., Kuehl, F. A., Wightman, P. D., Dahlgren, M., Davies, P. \& Bonney, R. J. (1982). Evidence for two sources of archidonic acid for oxidative metabolism by mouse peritoneal macrophages. Journal of Biological Chemistry 257, 1591-1594. 
Kunkel, S. L. \& Chensue, S. W. (1985). Archidonic acid metabolites regulate interleukin-1 production. Biochemical and Biophysical Research Communications 128, 892-897.

Levine, R. P. (1982). How is the level of free arachidonic acid controlled in mammalian cells? Biochemical Journal 204, 3-16.

Mead, C. J., Harvey, J., Boot, J. H., Turner, G. A., Bateman, P. E. \& Osborne, D. J. (1984). Hexachlorocyclohexane stimulation of macrophage phospholipid hydrolysis and leukotriene production. Biochemical Pharmacology 33, 289-293.

Ophir, R., Ben-Efraim, S. \& Bonta, I. (1987). Leukotriene $D_{4}$ and indomethacin enhance additively the macrophage cytostatic activity in vitro towards MOPC-315 tumour cells. International Journal of Tissue Reactions IX, 189-194

Schenkelaars, E. J. \& Bonta, I. L. (1986). Cyclooxygenase inhibitors promote the leukotriene $\mathbf{C}_{4}$ induced release of $\beta$-glucuronidase from rat peritoneal macrophages: prostaglandin $\mathrm{E}_{2}$ suppresses. International Journal of Immunopharmacology 8, 305-311.

Schinetti, M. L. \& Mazzini, A. (1986). Effects of L-carnitine on human neutrophil activity. International Journal of Tissue Reactions VIII, 199-203.

Smith, R. L. \& Weidemann, M. J. (1980). Reactive oxygen production associated with arachidonic acid metabolism by peritoneal macrophages. Biochemical and Biophysical Research Communications 97, 973-980.

Vargaftig, B. B., Chignard, M., Mencia-Huerta, J. M., Arnoux, B. \& Benveniste, J. (1981). Pharmacology of arachidonic acid metabolites and of platelet-activating factor (PAF-acether). In Platelets in Biology and Pathology, pp. 373-406 [J. Gordon, editor]. Amsterdam: Elsevier/North-Holland Biomedical Press.

Wroblewsky, F. \& LaDue, J. S. (1955). Lactic dehydrogenase activity in blood. Proceedings of the Society for Experimental Biology and Medicine 20, 210-213.

Zijlstra, F. J. \& Vincent, J. E. (1984). Determination of leukotrienes and prostaglandins in $\left[{ }^{14} \mathrm{C}\right]$ arachidonic acid labelled human lung tissue by high-performance liquid chromatography and radioimmunoassay. Journal of Chromatography 311, 39-50. 